6 De Haes JCJM, van Knippenberg FCE, Neijt JP. Measuring psychological and physical distress in cancer patients; structure and application of the Rotterdam symptom checklist. Br J Cancer 1990;62:1034-8.

7 Ibbotson T, Maguire P, Selby P, Priestman T, Wallace L. Screening for anxiety and depression in cancer patients: the effects of disease and treatment Eur J Cancer 1994.30A.37-40.

8 Fallowfield LJ, Hall A, Maguire GP, Baum M. Psychological outcomes of different treatment policies in women with early breast cancer outside a different treatment policies in women
clinical trial. $B M J$ 1990;301:575-80.

clinical trial. $B M J$ 1990;301:575-80.
Devlen J. Psychological and social morbidity in lymphoma patients $[\mathrm{PhD}$

thesis]. Manchester: University of Manchester 1984.
10 Pannuti F, Tannenberger S. The Bologna Eubiosia Project: hospital-athome care for advanced cancer patients. J Palliat Care 1992;8:11-7.

11 Hing E. Characteristics of elderly home health patients: preliminary data from the 1992 national home and hospice care survey. National Center for Health Statistics, 1994

12 Bonnema J, van Wersch AMEA, van Geel AN, Pruyn JFA, Schmitz PIM, Paul MA, et al. Medical and psychosocial effects of early discharge after surgery for breast cancer: randomised trial. BMJ 1998;316:1267-70.

(Accepted 26 June 1998)

\title{
One day survey by the Mental Health Act Commission of acute adult psychiatric inpatient wards in England and Wales
}

\author{
Richard Ford, Graham Durcan, Lesley Warner, Pollyanna Hardy, Matt Muijen
}

\begin{abstract}
Objectives To provide (via the Mental Health Act Commission's "national visit") empirical evidence on ward occupancy levels, use of the Mental Health Act 1983, nurse staffing, and care of female patients on acute adult psychiatric wards.

Design One day survey of a stratified random sample. Settings 119/250 (47\%) acute adult psychiatric inpatient units in England and Wales.

Subjects End sample of 263 acute psychiatric inpatient wards.

Main outcome measures Ward occupancy rates; number of patients detained under the Mental Health Act and proportion "absent without leave"; nurse staffing levels, skill mix, and vacancies; proportion of women with self contained, women-only facilities.

Results Mean ward occupancy was 99\% (95\% confidence interval $97 \%$ to $102 \%$ ). A ward mean of $30 \%(28 \%$ to $32 \%)$ of patients were detained under the Mental Health Act; of all detained patients, $1 \%$ ( $1 \%$ to $2 \%$ ) were absent without leave. A ward mean of $0.3(0.29$ to 0.31$)$ nurses were on duty per patient at the time of the visit. An estimated ward mean of $31 \%$ $(30 \%$ to $32 \%)$ of nurse staffing may have been through casual contracts-higher in inner (48\% (43\% to $53 \%)$ ) and outer London ( $45 \%$ (41\% to $48 \%))$. On $26 \%(21 \%$ to $32 \%)$ of wards, there were no nurses interacting with patients. A ward mean of 36\% (30\% to $41 \%$ ) of female patients had self contained, women-only facilities.
\end{abstract}

Conclusions Attention should focus on improving the quality of acute inpatient psychiatric care as well as of community care.

\section{Introduction}

Over the past 50 years the number of psychiatric inpatient beds in England has decreased greatly. The number has fallen from a peak of over 150000 in England in 1955 to 42000 in 1994-5. ${ }^{1}$ Despite the increase in community services, these beds still account for two thirds of expenditure on mental health services, ${ }^{2}$ and they remain an essential and large component of the range of services for severe mental illness. ${ }^{3}$ Despite the fall in overall bed numbers, the number of admissions a year has increased over the past decade from 207000 in 1984 to 237000 in 1994-5, representing an increase in throughput per bed each year, from 2.6 to 5.7 .

Acute inpatient services are reported to be under considerable pressure. ${ }^{4}$ The number of formal admissions increased by 53\% over five years, to 26100 in 1994-5. At the same time, some former inpatients on acute psychiatric wards have commented on the poor quality of care they received. ${ }^{6}$

On 21 November 1996 the Mental Health Act Commission, in collaboration with the Sainsbury Centre for Mental Health, made an unannounced visit to acute psychiatric wards in England and Wales. The innovative approach of a "national visit" was seen as a new and additional way for the commission to pursue its statutory responsibilities.

The aims of the national visit were identified by the commission in previous reports on matters of concern $^{78}$ : to investigate further the level of pressure on acute admission wards; to investigate the extent to which patients were going absent from the wards while still legally detained; to evaluate the level of nursing input to care; and to examine the appropriateness of ward facilities for the safe care of female patients. This paper reports how the visit was conducted and its findings.

\section{Methods}

\section{Sampling}

A list of all NHS trusts providing acute inpatient psychiatric care to people aged 16 to 64 years was obtained from the NHS year book $1995-6 .{ }^{9}$ Trusts were included if $(a)$ they had wards that were designated as acute; (b) most patients were in hospital for less than three months; and $(c)$ most patients were aged 18 to 65 . Wards designated as intensive care were included; medium secure units, regional secure units, special hospitals, the independent sector, and acute units providing only a "mother and baby" service were excluded. All the trusts on the list that were not known to the research team were telephoned so that details could be checked. Each of the Mental Health Act Commission's

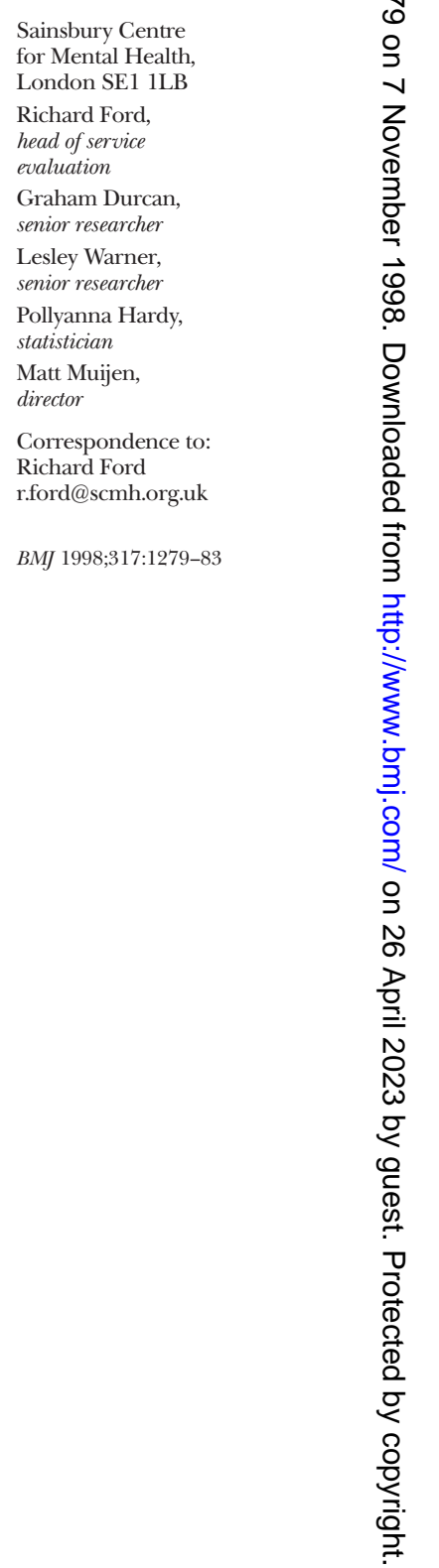


seven regional visiting teams was sent a list of wards in its region to ensure that all appropriate wards were included. Two stage, stratified random sampling was used. Firstly, 17 NHS trusts were randomly selected for each regional visiting team; these represented $62 \%$ $(119 / 192)$ of NHS trusts with acute psychiatric facilities across England and Wales. Secondly, where a trust had more than one geographical base, one unit was randomly selected for the national visit; this represented 47\% (119/250) of all acute inpatient units. Every ward was visited for each selected unit. The sample size was determined by the number of commissioners (staff from the Mental Health Act Commission) available to make visits.

\section{Instruments}

The specific issues to be addressed by the national visit were identified by the commission, which organised a steering group of chief officers and regional convenors. The research team from the Sainsbury Centre for Mental Health met the steering group on many occasions to agree the proforma that commissioners would complete. Each version of the proforma was amended in the light of piloting work (20 versions in total). At the same time, a detailed instruction booklet was compiled to help commissioners. The research team then trained the regional convenors to use the proforma, and the research team and regional convenors then trained all commissioners taking part in the national visit.

\section{Procedure}

The national visit was conducted on a single day, 21 November 1996. All available commissioners were told by the convenor of their regional visiting team which units to visit. The visits were undertaken during day shifts by commissioners working in pairs, using their powers to make unannounced visits. In this way, none of the wards visited was informed of the visits in advance. The commission staffed a helpline for commissioners to overcome any difficulties with access, and the research team staffed a helpline to deal with any difficulties in interpreting the proforma. In the event, no difficulties with access arose, and the research team responded to only 12 minor calls.

Analysis

For this article the analysis was restricted to the 263 wards clearly identified by commissioners as being acute admission wards at the time of the visit. A further 33 intensive care wards were visited, and 13 of the returned proformas did not record the type of ward. Proportions, means, standard deviations, and 95\% confidence intervals (using exact methods for proportions) are reported as appropriate. After the proformas were returned, each of the units visited was classified according to the type of local authority within which it was situated. Local authority boundaries as at March 1996 were used to classify each unit as being in inner London, outer London, a metropolitan area, a county council area, or Wales. For continuous data, when approximately normally distributed, comparisons were made between means for the local authority types by using univariate analyses of variance and least significant difference tests. For categorical data, differences were analysed with $\chi^{2}$ tests. All tests were two sided, and $\mathrm{P}$ values of $<0.05$ were considered significant. The data were analysed with the statistical software sPss for Windows version 6.1 and STATA version 5.0

\section{Results}

On the day of the national visit, all units except one (which was snowed in) were visited, giving an end sample of $118(47 \%)$ acute psychiatric units. Owing to sickness or other unforeseen events, 22 of the 263 wards were visited by only one commissioner, rather than two. In all, 15 wards were in inner London, 37 in outer London, 55 in metropolitan areas, 145 in county council areas, and 11 in Wales.

\section{Occupancy}

The sample comprised 5971 beds (mean number per ward 22.7 (95\% confidence interval 22.0 to 23.4$)$ ), with no variation by local authority type $(\mathrm{F}=0.99 ; \mathrm{df} 4,258$; $\mathrm{P}=0.4$ ). Occupancy of these beds varied by authority type, with inner and outer London having significantly higher levels (table 1). After the number of patients on overnight leave was deducted from the occupancy figure, however, no variation by authority type was seen. The proportion of patients (excluding those on overnight leave) who were women did not seem to vary by authority type. The proportion of patients (including those on overnight leave) who were detained under a section of the Mental Health Act 1983 at the time of the visit was higher in both inner and outer London than elsewhere in England and Wales.

Table 1 Ward means (95\% confidence intervals) for percentage occupancy, sex, and percentage of patients detained under the Mental Health Act, by type of local authority

\begin{tabular}{|c|c|c|c|c|}
\hline \multirow[b]{2}{*}{ Local authority (No of wards) } & \multicolumn{2}{|c|}{$\%$ occupancy } & \multirow[b]{2}{*}{$\%$ of women } & \multirow[b]{2}{*}{$\%$ of patients detained } \\
\hline & $\begin{array}{l}\text { Including patients on } \\
\text { overnight leave* }\end{array}$ & $\begin{array}{l}\text { Excluding patients on } \\
\text { overnight leave }\end{array}$ & & \\
\hline Inner London $(\mathrm{n}=15)$ & 107 (91 to 123) & 86 (76 to 96$)$ & 53 (41 to 65$)$ & 44 (36 to 52$)$ \\
\hline Outer London $(\mathrm{n}=37)$ & 111 (105 to 117$)$ & 93 (87 to 98$)$ & 48 (44 to 53$)$ & 34 (29 to 38$)$ \\
\hline Metropolitan $(n=55)$ & 93 (90 to 97$)$ & 84 (80 to 88$)$ & $51(46$ to 56$)$ & $27(23$ to 30$)$ \\
\hline County council $(n=145)$ & 98 (96 to 101$)$ & 85 (82 to 88$)$ & 54 (51 to 57$)$ & 29 (27 to 31$)$ \\
\hline Wales $(n=11)$ & 92 (76 to 107$)$ & 82 (66 to 99) & 53 (43 to 64$)$ & 27 (17 to 38$)$ \\
\hline Total $(n=263)$ & 99 (97 to 102) & 86 (84 to 88$)$ & 53 (50 to 55$)$ & 30 (28 to 32$)$ \\
\hline Significance (F test) & $F=6.68 ;$ df 4,$258 ; P<0.001$ & $F=2.13 ;$ df 4,$257 ; P=0.077$ & $F=0.95 ;$ df 4,$253 ; P=0.435$ & $F=5.35 ;$ df 4,$258 ; P<0.001$ \\
\hline
\end{tabular}

${ }^{*}$ Number of patients/number of beds.

†(Number of patients minus number on overnight leave)/number of beds. 
Table 2 Ward means (95\% confidence interval) for nurse staffing levels, by type of local authority

\begin{tabular}{|c|c|c|c|c|c|c|}
\hline \multirow[b]{2}{*}{ Local authority } & \multicolumn{2}{|c|}{ No of staff needed per patient } & \multirow{2}{*}{$\begin{array}{l}\text { Estimated No of } \\
\text { vacancies } \dagger\end{array}$} & \multirow{2}{*}{$\begin{array}{l}\text { No of staff a trust } \\
\text { aims to employ }\end{array}$} & \multirow{2}{*}{$\begin{array}{c}\% \text { of regular staff } \\
\text { on duty }\end{array}$} & \multirow{2}{*}{$\begin{array}{c}\% \text { of qualified staf } \\
\text { on duty }\end{array}$} \\
\hline & Estimated $^{*}$ & Actual & & & & \\
\hline Inner London $(\mathrm{n}=15)$ & 1.66 (1.37 to 1.95$)$ & $0.86(0.71$ to 1.01$)$ & 13.01 (9.21 to 16.80$)$ & $1.19(0.98$ to 1.4$)$ & 90 (84 to 96$)$ & 78 (70 to 86$)$ \\
\hline Outer London $(\mathrm{n}=37)$ & $1.40(1.24$ to 1.56$)$ & 0.77 (0.66 to 0.87$)$ & 12.41 (10.29 to 14.52) & $0.97(0.86$ to 1.0$)$ & 88 (81 to 94$)$ & 63 (58 to 68$)$ \\
\hline Metropolitan $(n=55)$ & $1.36(1.23$ to 1.50$)$ & 1.07 (0.96 to 1.18$)$ & 5.87 (3.76 to 7.98$)$ & 1.05 (0.94 to 1.1$)$ & 96 (93 to 99) & 51 (47 to 56$)$ \\
\hline County council $(n=145)$ & 1.50 (1.40 to 1.60$)$ & 1.09 (1.01 to 1.17$)$ & 7.53 (6.31 to 8.75$)$ & 1.17 (1.09 to 1.2$)$ & 94 (91 to 96$)$ & 61 (58 to 64$)$ \\
\hline Wales $(n=11)$ & 1.51 (1.03 to 1.99$)$ & 0.95 (0.66 to 1.24) & 8.98 (5.87 to 12.10$)$ & 0.93 (0.65 to 1.2$)$ & 96 (91 to 102) & 65 (57 to 73$)$ \\
\hline Total $(n=263)$ & 1.47 (1.40 to 1.54$)$ & 1.02 (0.96 to 1.07$)$ & 8.24 (7.32 to 9.16$)$ & $1.11(1.05$ to 1.1$)$ & 93 (91 to 95$)$ & 61 (59 to 63$)$ \\
\hline
\end{tabular}

${ }^{*}$ Actual staff on duty multiplied by 5.1 for non-handover and 3.6 for handover period. ${ }^{10}$

†Estimated number of staff needed minus actual number of permanent staff.

Table 3 Ward percentage means (95\% confidence intervals) for women with access to each category of facility, by type of local authority

\begin{tabular}{|c|c|c|c|c|}
\hline Local authority & Category A & Category B & Category C & Category D \\
\hline Inner London $(\mathrm{n}=15)$ & 53 (25 to 81$)$ & 27 (3 to 51) & 18 (0 to 39$)$ & 0 \\
\hline Outer London $(n=37)$ & 30 (15 to 44$)$ & 40 (24 to 55$)$ & 31 (16 to 45$)$ & 0 \\
\hline Metropolitan $(n=55)$ & $38(26$ to 50$)$ & $32(20$ to 44$)$ & 30 (18 to 42$)$ & 0 \\
\hline County council $(n=145)$ & 31 (24 to 38 ) & 24 (17 to 31$)$ & 40 (32 to 48$)$ & 5 (2 to 9$)$ \\
\hline Wales $(n=11)$ & 81 (54 to 108$)$ & $19(0$ to 46$)$ & 0 & 0 \\
\hline Total $(n=263)$ & 36 (30 to 41$)$ & 28 (23 to 33 ) & 33 (28 to 39 ) & $3(1$ to 5$)$ \\
\hline
\end{tabular}

Category $A=$ self contained, with women-only amenities; category $B=$ separate sleeping areas, with access to separate amenities via mixed areas; category $C=s e p a r a t e$ sleeping areas, with amenities for mixed use; category D=mixed sleeping areas. All women patients (excluding those on leave) were assigned to one of categories $A$, B, C, or D. "Amenities" comprises baths, showers, and toilets.

\section{Nurse staffing}

The commissioners found a mean of 0.3 (0.29 to 0.31$)$ nursing staff on duty for every patient (excluding those on overnight leave). No variation by authority type was discernible $(\mathrm{F}=0.72$; df 4,$257 ; \mathrm{P}=0.6)$. Time of visit was not associated with the number of staff on duty (Spearman's $r=0.03, \mathrm{P}=0.7$ ). Few visits took place during the nursing staff's handover periods (28/259; $11 \%(7 \%$ to $15 \%))$, although more staff were on duty then than at non-handover times (mean per patient 0.41 (0.35 to 0.47$) v 0.29$ ( 0.27 to 0.32$) ; t=5.28$; df 256 ; $\mathrm{P}<0.001)$. An estimated ward mean of 1.47 whole time equivalent staff per patient (table 2) would need to be employed to give this level of staffing (staff on duty multiplied by 5.1 for non-handover and 3.6 for handover period $\left.^{10}\right)$. In fact, there was a ward mean of 1.02 whole time equivalent staff currently employed per patient, which varied by authority type, being lower in inner and outer London. It can therefore be estimated that there were vacancies for a mean of 8.25 whole time equivalent staff, which were being filled by staff not on a permanent contract with the NHS trust. This vacancy rate varied by authority type, with both inner and outer London having higher rates. It can be further extrapolated that 4524/14 368 (31\% (30\% to $32 \%)$ ) whole time equivalent posts across England and Wales were being filled by staff not working on a permanent contract with their trust (inner London, $410 / 854$ (48\%; $43 \%$ to $53 \%)$; outer London, $965 / 2144$ $(45 \% ; 41 \%$ to $48 \%))$.

An alternative way of investigating vacancy rates would be to consider the difference between the "set staff establishment" (the number of staff a trust aims to employ) and the number of staff currently in post. This method was not used as the reported levels of set staff establishment seemed to be unreliable. For example, this method shows that metropolitan authorities and Wales had on average more staff in post than the mean set staff establishment would allow (table 2).
The commissioners found that a ward mean of $93 \%$ of staff worked regularly (at least four shifts on the ward in the past two weeks), with no significant variation by authority type. They also found that a ward mean of $61 \%$ of the nursing staff had a qualification, which varied by authority type, with inner London having the highest proportion and metropolitan areas the lowest.

When the commissioners arrived at the ward they asked to be shown where every nurse was. Commissioners found that there was no nurse interacting with patients on $69 / 262(26 \%(21 \%$ to $32 \%))$ wards, with no variation by authority type $\left(\chi^{2}=4.84\right.$; df $\left.4 ; \mathrm{P}=0.3\right)$. Commissioners then asked if nurses were involved in special observation: one or more members of nursing staff were carrying out continuous observation of one or more patients on $88 / 262(34 \% ; 28 \%$ to $40 \%)$ wards or frequent observation of one or more patients on $154 / 262(60 \% ; 53 \%$ to $65 \%)$ wards; on some wards nurses were involved in both of these activities. Over the whole sample, $30 / 261(11 \%(8 \%$ to $16 \%))$ wards had a member of staff permanently stationed at the door of the ward to control the exit of patients. Such "door duty" varied $\left(\chi^{2}=10.55\right.$; df $\left.4 ; \mathrm{P}=0.03\right)$, from $0 / 11(0 \%(0 \%$ to $28 \%))$ wards in Wales, through $1 / 54$ $(2 \%(0 \%$ to $10 \%))$ in metropolitan areas, $19 / 144(13 \%$ $(8 \%$ to $20 \%))$ in county council areas, and $2 / 15(13 \%$ (2\% to $40 \%))$ in inner London, to $8 / 37(22 \%(10 \%$ to $38 \%)$ ) in outer London.

\section{Facilities for women}

Table 3 shows that a ward mean of $36 \%$ of women had access to self contained facilities where they could sleep and have access to amenities (that is, showers, baths, and toilets) without male patients having any access. This proportion varied significantly by local authority type: wards in Wales were most likely to have such facilities and those in county council areas least likely. There was no variation by local authority type for 
the proportion of women (ward mean 28\%) who had separate sleeping areas but could reach amenities only by going through areas to which male patients had access. The proportion of women who had to share amenities (ward mean 33\%), however, varied significantly by local authority type, with Wales having no wards in this category. A ward mean of $3 \%$ of women shared sleeping areas with men (no significant variation by local authority type).

\section{Security issues}

On arrival, commissioners found 23/261 (9\% (6\% to $13 \%)$ ) wards to be locked so that patients could not leave without asking a member of staff to unlock the door. Commissioners asked if any patients who were detained under the Mental Health Act were "absent [from the ward] without leave." Commissioners had been asked to check whether all detained patients who were absent had had their leave authorised by the responsible medical officer under section 17 of the act. Altogether, $47 / 206$ (23\% (17\% to $29 \%))$ wards had patients who were absent without leave (45 wards with one patient and 2 wards with two patients). Several of these patients, however, had been recorded by commissioners as absent without leave only because some wards' paper work was not properly completedfor example, no written record of leave granted by the responsible medical officer. After closer examination, 26 patients on 24 wards were considered to be absent without leave, out of 1752 detained patients ( $1 \%$ ( $1 \%$ to $2 \%)$ ). There was no relation between the ward being unlocked and patients being absent without leave $\left(\chi^{2}=0.08 ;\right.$ df $\left.1 ; \mathrm{P}=0.8\right)$. There was no significant variation by authority type for any of the security issues reported here.

\section{Discussion}

The Mental Health Act Commission's national visit has confirmed previous reports of high bed occupancy (99\%), especially in London (inner 109\%, outer 111\%). However, after patients on leave were discounted, the mean occupancy level fell to $86 \%$ and was no higher in London than elsewhere. Anecdotally, periods of leave seem to be authorised routinely for detained patients as a mechanism for allowing trial periods in the community with the option of immediate recall to hospital if necessary. Psychiatrists would have to readmit to hospital formally under the Mental Health Act 1983 (even as amended for supervised discharge) if these patients were discharged. This practice is probably more common in inner London, with a higher proportion of patients being detained under section than in the rest of England and Wales. This study shows that acute inpatient facilities may be under substantial pressure but that some of this pressure derives from bed management difficulties stemming from the use of leave for detained patients. Often periods of leave are for short or unknown periods of time, with the patient likely to return at any time without warning. Such beds are not, therefore, easy to use for other patients requiring admission. Other factors, such as the lack of suitable accommodation for patients ready for discharge, ${ }^{4}$ also contribute to the pressure on acute psychiatric beds.
- Leave arrangements on adult psychiatric wards cause considerable difficulties in bed management

- An estimated third of nursing staff on duty are employed on a casual basis

- Nurses spend much of their time engaged in intensive observation of a few patients, but a quarter of wards had no nurse interacting with patients at the time of the national visit

- Only a third of female patients had the use of self contained, women-only areas

- Policymaking, management, and training must be refocused to improve the quality of acute inpatient psychiatric care

A high proportion of vacancies was being filled by staff not working to a permanent contract for that shift, though the vast majority of these non-permanent staff were working on the particular ward on a regular basis. The estimated vacancy rate of $28 \%$ (higher in London) was probably being filled by staff working either for an agency or for a bank system operated by the managing NHS trust. Often such staff also have a permanent contract with the trust and use agency or bank working as a source of overtime. For the trust, there can be cost savings as it does not have to pay premium overtime rates or for sickness, annual leave, or training. This high vacancy rate is largely hidden as the staffing levels that trusts aim to achieve are lower than the levels of actual staffing and do not adequately reflect the staffing levels needed. The proportion of nursing staff on the ward holding a formal qualification was not consistentranging from $18 \%$ in metropolitan areas to $47 \%$ in inner London. Without information on patients' needs, we cannot say whether staffing levels or skill mix were appropriate. $^{11}$

On a quarter of wards there was no nurse in contact with patients at the time of the visit. At the same time, considerable numbers of nurses were engaged in continuous observation, frequent observation, and door duty, but not necessarily direct contact with patients. Wards also had problems implementing some of the procedures of the Mental Health Act, such as section 17 . However, given that the vast majority of wards were unlocked, few patients $(1 \%)$ were absent without leave. These findings, taken in conjunction with patients' reports of boredom and not enough contact with professional staff ${ }^{72}$ and reports from inquiries showing that nurses were not aware of the whereabouts of their patients, ${ }^{13}$ raise serious issues.

Female patients can often feel vulnerable on an acute psychiatric ward. The need for safe, women-only wards or areas on wards has been recognised. ${ }^{14}$ However, it is not clear how this objective can be achieved without considerable restructuring and additional resources, given that only a third of wards reached the desired standard (trusts in Wales would have less difficulty in creating safe facilities for women on all psychiatric wards). 


\section{Conclusions}

The combination of increasingly pressured acute wards and high levels of casual staffing cannot be good for the care of patients. These problems are most severe in London, where patients have more severe problems, as evidenced by the higher proportion of patients on the ward who are detained under the Mental Health Act. The policy, managerial, and training focus has been on developing community services. This has resulted in a relative neglect of inpatient settings. The national visit has shown that attention must be given to inpatient wards, which are an essential and major element of mental health care.

Contributors: RF led discussions with the Mental Health Act carried out analysis of the data. GD and LW helped with the study design, piloted methods, trained commissioners, and carried out analysis of the data. PH assisted with the study design, advised on all analysis and carried out analysis. This paper was written jointly by all authors. RF is guarantor for this paper.

Funding: The Mental Health Act Commission funded the design, methods, analysis, and reporting.

Conflict of interest: None. Commission, initiated the study design (along with MM), and national visit, and the Gatsby Charitable Trusts funded the
1 Department of Health. Health and personal social services statistics for England. London: Stationery Office, 1996

2 Audit Commission. Finding a place: a review of mental health services for adults. London: HMSO, 1994.

3 Department of Health. The spectrum of care: local services for people with mental health problems. Wetherby: $\mathrm{DoH}, 1996$.

4 Shepherd G, Beadsmoore A, Moore C, Hardy P, Muijen M. Relation between bed use, social deprivation, and overall bed availability in acute adult psychiatric units, and alternative residential options: a cross sectional survey, one day census data, and staff interviews. $B M$ J 1997;314:262-6.

5 Department of Health. In-patients formally detained in hospitals under the Mental Health Act 1983 and other legislation: 1989-90 to 1994-95. London: Stationery Office, 1996. (Statistical bulletin 1996/10.)

6 Moore C. Acute in-patient care could do better, says survey. Nursing Times 1998;94(3):54-6.

7 Mental Health Act Commission. Sixth biennial report 1993-95. London: HMSO, 1995.

8 Mental Health Act Commission. Deaths of detained patients. London Mental Health Foundation, 1995.

9 Howland G, ed. The IHSM health and social services yearbook 1996/97. London: Institute of Health Services Management, 1996.

10 Hurst K. Promotions and relegations in the psychiatric nursing league. J Nurs Manage 1995;3:43-6.

11 Royal College of Psychiatrists. Not just bricks and mortar: a report of the Royal College of Psychiatrists Working Party on the size, staffing, structure $\mathcal{E}$ siting and security of new acute adult inpatient units. London: RCP, 1997.

12 Mental Health Act Commission. Seventh biennial report 1995-97. London: Stationery Office, 1997.

13 Blom-Cooper L, Hally H, Murphy E. The falling shadow-one patient's mental health care 1978-1993. London: Duckworth, 1995.

14 Snell J. Mixed is no match. Health Service Journal 1997;107(5573):26-9.

(Accepted 28 July 1998)

\section{Suicide, deprivation, and unemployment: record linkage study}

\author{
Glyn Lewis, Andy Sloggett
}

\begin{abstract}
Objectives To investigate the association between suicide and socioeconomic status, unemployment, and chronic illness.

Design Longitudinal study.

Setting England and Wales.

Subjects Individuals from the Office for National Statistics longitudinal study for whom 1981 census data were available. The longitudinal study is a representative $1 \%$ sample of the population of England and Wales in which census variables are linked to mortality data.
\end{abstract}

Main outcome measures Suicide and undetermined deaths occurring between 1983 and 1992. Odds ratios estimated with logistic regression adjusted for attrition of cohort members.

Results There was a strong independent association between suicide and individuals who were unemployed (odds ratio 2.6; 95\% confidence interval 2.0 to 3.4$)$ and permanently sick $(2.5 ; 1.6$ to 4.0$)$. Those without access to a car had an increased risk (1.3; 1.0 to 1.5$)$, but other measures of socioeconomic status were not associated with suicide.

Conclusions The association between suicide and unemployment is more important than the association with other socioeconomic measures. Although some potentially important confounders were not adjusted for, the findings support the idea that unemployment or lack of job security increases the risk of suicide and that social and economic policies that reduce unemployment will also reduce

\section{Introduction} numbers of premature deaths, and to inform policies that might prevent suicide there is a need for improved knowledge about risk factors. Previous studies have used data from death certificates to investigate the relation between registrar general's social class and suicide. Before the second world war there was an inverse gradient of social class with higher rates in social classes I and II than IV and V. ${ }^{1}$ Subsequent findings have described a "U shaped" distribution with higher rates in both social classes I and $\mathrm{V},{ }^{12}$ though this may be a cohort effect apparent only in older age groups." The most recent studies have found higher rates in lower social classes, though the relation does not look linear over the whole gradient of social class. ${ }^{3}$

There are also other variables that relate to socioeconomic status including educational attainment and standard of living, where housing tenure and access to a car can be used as indicators. ${ }^{45}$ These have been little studied in relation to suicide. There is evidence that the availability of methods for suicide can influence rate of suicide $\mathrm{e}^{67}$ and poisoning with car exhaust fumes is becoming an increasingly common method. ${ }^{8}$ It is therefore possible that access to a car would increase the risk of suicide after adjustment for other socioeconomic differences between people. the rate of suicide.

Suicides make a substantial contribution to the

Division of
Psychological
Medicine,
University of Wales
College of
Medicine, Cardiff
CF4 4XN
Glyn Lewis,
professor of
community and
epidemiological
psychiatry 